\title{
Prognosis and management of new-onset atrial fibrillation in critically ill patients
}

\author{
Jun Qian' ${ }^{1}$ Lijun Kuang ${ }^{2}$, Fei Chen ${ }^{1}$, Xuebo Liu ${ }^{1 *}$ and Lin Che ${ }^{1 *}$
}

\begin{abstract}
Introduction: The prognosis of new-onset atrial fibrillation (AF) compared with that of preexisting and non-AF remains controversial. The purpose of this study was to evaluate the effect of new-onset AF compared with preexisting and non-AF on hospital and 90-day mortality.

Methods: A retrospective cohort study was performed using data obtained from the Medical Information Mart for Intensive Care III database. The primary outcome was 90-day mortality. Secondary outcomes included hospital mortality, hospital and intensive care unit (ICU) length of stay, and acute kidney injury. Logistic and Cox regression analyses were performed to evaluate the relationship between new-onset AF and study outcomes.

Results: A total of 38,159 adult patients were included in the study. The incidence of new-onset AF was $9.4 \%$. Ninety-day mortality, hospital mortality, and hospital and ICU length of stay in patients with new-onset and preexisting AF were significantly increased compared with those in patients with non-AF patients (all $p<0.001$ ). After adjusting for patient characteristics, new-onset AF remained associated with increased 90-day mortality compared with non-AF (adjusted hazard ratio (HR) 1.37, 95\% confidence interval (Cl) 1.26 to 1.50; $p<0.01$ ) and preexisting AF (adjusted HR 1.12; 95\%-Cl 1.02 to $1.23 ; \mathrm{p}<0.01$ ). Patients in the surgical intensive care unit (SICU) had significantly higher 90-day mortality than patients in the coronary care unit (adjusted HR 1.30; 95\% Cl 1.31 to 1.51; $\mathrm{p}<0.001$ ).
\end{abstract}

Conclusions: Critically ill patients with new-onset AF have significantly increased hospital and 90-day mortality compared with patients with preexisting and non-AF. Patients with new-onset AF in the ICU, especially those in the SICU, require robust management measures.

Keywords: Atrial fibrillation, Critical care, Mortality, Intensive care unit

\section{Background}

Atrial fibrillation (AF) may present as irregular, rapid, electrical and mechanical activation of the atria, resulting in asynchronous contraction of the atria that may promote thromboembolism formation [1]. AF is the most common arrhythmia in clinical settings, especially in intensive care unit (ICU) patients [2]. AF is mainly divided into paroxysmal AF, persistent $\mathrm{AF}$, long-term

\footnotetext{
*Correspondence: liuxb70@126.com; linchering@163.com

${ }^{1}$ Department of Cardiology, Shanghai Tongji Hospital, Tongji University School of Medicine, No.389, Xincun Rd, putuo District, Shanghai 200065, China

Full list of author information is available at the end of the article
}

persistent AF, and permanent AF [3]. Preexisting AF is very common among ICU patients, while new-onset $A F$ is also a frequent complication in the ICU with an incidence of approximately $5 \%$ [4-6].

Causes of new-onset AF might include electrolyte disturbances, fluid imbalances, neurohormonal disturbances, arrhythmic drug use, and inflammatory reactions [4]. Chronic heart failure, hypertension, valvular disease, and myocardial infarction trigger a variety of common inflammatory pathways, activation of the renin-angiotensin system and production of reactive oxygen species that lead to atrial fibrosis and further promote the occurrence of AF [7]. In patients who have undergone surgery and had a recent myocardial 
infarction, AF was often associated with poor outcomes, such as an increased risk of stroke [8-12]. However, evidence of the effects of new-onset AF, preexisting and non-AF with prognosis in ICU patients is limited and contradictory.

Preexisting and new-onset AF have been shown to be associated with all-cause mortality [13, 14]. However, several studies have shown that preexisting and newonset AF may not be independently associated with hospital mortality $[15,16]$. A cohort study including more than 1300 critically ill patients with persistent arrhythmias found that AF was not associated with increased mortality [17]. Many previous studies had limited sample sizes, and their conclusions were controversial. The aim of this study was to identify the associations of new-onset $\mathrm{AF}$, preexisting AF, and non-AF with hospital and 90-day mortality among ICU. This retrospective observational study was performed in accordance with the STROBE reporting checklist.

\section{Methods}

\section{Database access}

We performed a retrospective study based on the 'Medical Information Mart for Intensive Care (MIMIC) III' database [18]. The database includes comprehensive clinical information of inpatients treated at the Beth Israel Deaconess Medical Center (BIDMC) in Boston, Massachusetts from June 1, 2001 to October 31, 2012. The use of this database was approved by the BIDMC Institutional Review Board. Our data extraction was performed by Dr. Qian who obtained access to the database (certificate code: 32,299,459). https://mimic.physionet.org/getti ngstarted/access/.

\section{Patient and data extraction}

The exclusion criteria were age younger than 18 years and death before hospitalization. All other patients $(\geq 18$ years) alive at admission were included in the study. For patients who were admitted multiple times, only the data from first ICU admission was retained. The detailed research flow chart is shown in Fig. 1. Information extracted from the database included age, gender, comorbidities on admission, type of ICU, laboratory tests performed on admission, Sequential Organ Failure Assessment (SOFA) score, medications during hospitalization and length of stay (LOS). The type of ICU each patient was admitted to was determined by the physician based on pathological state of each patient at the time of admission. All drugs referred to in our study were administered at the time of admission and continued after discharge.

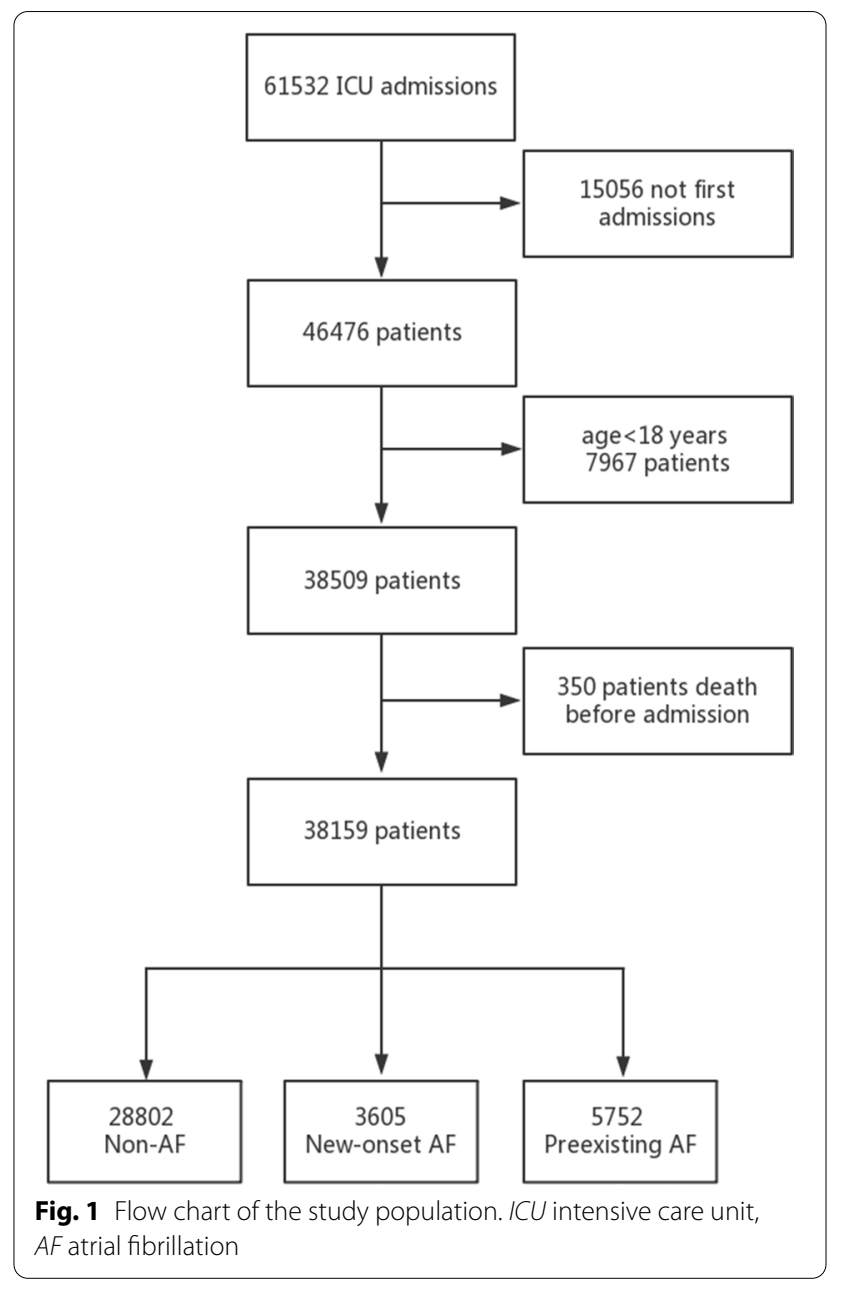

\section{Definitions and outcomes}

We divided all patients into three groups: non-AF, newonset $\mathrm{AF}$, and preexisting $\mathrm{AF}$. New-onset $\mathrm{AF}$ was defined as the first diagnosis during hospitalization based on a 12-lead electrocardiogram. Patients with a diagnosis of AF before hospital admission were identified as preexisting AF. Patients with neither AF diagnosis were defined as non-AF. The primary outcome was 90 -day mortality. Secondary outcomes included hospital mortality, hospital and ICU LOS, and acute kidney injury during hospitalization. Acute kidney injury was defined as a serum creatinine (Scr) level that was 1.5 times higher than baseline [19].

\section{Statistical analysis}

Continuous variables are expressed as the mean \pm standard deviation (SD) or median (interquartile range) as appropriate and were compared by analysis of variance or the Mann-Whitney $U$ test. Categorical variables are presented as percentages and were, compared by the chisquare test. The univariate Kaplan-Meier method was 
used to estimate the 90-day mortality among the three groups. Ninety-day and hospital mortality were assessed using a Cox regression model and a logistic regression model. We used two different models to adjust for potential confounders: (1) model 1, which included age, gender, type of ICU, and comorbidities on admission including hypertension, diabetes, coronary heart diseases, congestive heart failure, hyperlipidemia, chronic obstructive pulmonary disease (COPD), cerebral infarction, pulmonary embolism, sepsis, and hypothyroidism and (2) model 2, which included the SOFA score, laboratory tests performed on admission including the white blood cell (WBC) count, hemoglobin (HB) level, Scr, and medications administered during hospitalization including $\beta$ blockers, statins, amiodarone, non-dihydropyridine calcium channel blockers (CCB), digoxin, and warfarin in addition to the above mentioned variables from model 1 . The significance level was set at 0.05 , and all analyses were two-sided. Statistical analyses were performed using Statistical Package for the Social Sciences (SPSS) 22.0 (SPSS Inc., Armonk, NY, USA) and R software (version 3.6.1; http://www.R-project.org, R Foundation for Statistical Computing, Vienna, Austria).

\section{Results}

\section{Patients characteristics}

A total of 38,159 patients were enrolled in the study. The three groups of non-AF, new-onset $\mathrm{AF}$, and preexisting AF included 28,802, 3605, and 5752 patients, respectively. The incidence of new-onset AF during hospitalization was $9.4 \%$. Table 1 shows the baseline characteristics for the 3 groups. The age of patients with preexisting and new-onset AF was significantly older than that of patients with non-AF $(\mathrm{p}<0.001)$. Patients with new-onset AF had an increased prevalence of hypertension, diabetes, coronary heart disease, and hyperlipidemia. The prevalence of new-onset AF was the highest in the cardiac surgical recovery unit (CSRU). Patients with new-onset AF had the highest use of amiodarone and statins, while those with preexisting AF had the highest use of $\beta$ blockers, digoxin, and warfarin.

\section{Clinical outcomes}

Table 2 shows the unadjusted outcomes among the 3 groups. The 90-day mortality, hospital mortality, hospital and ICU LOS of patients with new-onset and preexisting AF were significantly increased compared with those of patients with non-AF (all $\mathrm{p}<0.001)$. Patients with nonAF had the highest proportion of acute kidney injury. The primary outcome of 90-day mortality among patients with non-AF, new-onset $\mathrm{AF}$, and preexisting $\mathrm{AF}$ was $15.26 \%, 20.83$ and $25.35 \%$, respectively. In patients with non-AF, new-onset AF, and preexisting AF the hospital mortality rate was $9.52 \%, 13.34$ and $14.97 \%$, respectively. Figure 2 shows the Kaplan-Meier curve for survival probability in the 3 groups, which is consistent with Table 2 . However, as presented in Table 3, the results of the multivariate Cox regression analyses show that new-onset AF was associated with an increased risk for 90-day mortality compared with non-AF and preexisting AF after adjustment using the two different models (model 2: compared with non-AF, adjusted hazard ratio (HR) 1.37, 95\% confidence interval (CI) 1.26-1.50, $\mathrm{p}<0.001$; compared with preexisting AF, HR 1.12, $95 \%$ CI 1.02-1.23, $\mathrm{p}=0.019$; model 1 is presented in Table 3). After adjusting two models using logistic regression analyses, newonset AF was also associated with an increased risk of hospital mortality compared with non-AF and preexisting AF, as shown Table 4 (model 2: compared with nonAF, HR 1.61, $95 \%$ CI 1.41-1.85, $\mathrm{p}<0.001$; compared with preexisting AF, HR 1.17, $95 \% \mathrm{CI} 1.01-1.35, \mathrm{p}=0.034$ ).

\section{Variables related to mortality}

Figure 3 shows the associations between different variables and 90-day mortality among patients with AF. After adjusting for the variables in model 2, we found that age (HR 1.03, 95\% CI 1.03-1.04, $\mathrm{p}<0.001$ ), congestive heart failure (HR 1.22, 95\% CI 1.11-1.33, $\mathrm{p}<0.001$ ), cerebral infarction (HR 1.78, 95\% CI 1.56-2.04, $\mathrm{p}<0.001$ ), pulmonary embolism (HR 1.51, 95\% CI 1.18-1.93, p<0.001), sepsis (HR 1.37, 95\% CI 1.22-1.55, $\mathrm{p}<0.001$ ), SOFA score (HR 1.15, 95\% CI 1.13-1.16, $\mathrm{p}<0.001$ ), WBC count (HR $1.02,95 \%$ CI 1.01-1.02, $\mathrm{p}<0.001$ ), amiodarone (HR 1.16, 95\% CI 1.05-1.29, $\mathrm{p}=0.004)$, non-dihydropyridine CCB use (HR 1.15, 95\% CI 1.03-1.27, $\mathrm{p}=0.011$ ), and digoxin (HR 1.23, 95\% CI 1.10-1.39, $\mathrm{p}<0.001$ ) were associated with a significantly increased risk of 90-day mortality. In contrast, hypertension (HR 0.86, 95\% CI 0.78-0.94, $\mathrm{p}=0.001$ ), hyperlipidemia (HR 0.82 , 95\% CI 0.72-0.92, $\mathrm{p}=0.001$ ), $\beta$-blockers use (HR 0.59, 95\% CI 0.53-0.65, $\mathrm{p}<0.001)$, statin use (HR 0.76, 95\% CI 0.68-0.86, $p<0.001$ ), and warfarin use (HR $0.42,95 \%$ CI $0.37-0.47$, $\mathrm{p}<0.001)$ were protective factors for 90 -day mortality in patients with AF. Patients with AF in the CSRU had a lower risk of 90-day mortality than patients with coronary care unit (CCU) (HR 0.37, 95\% CI 0.31-0.44, $\mathrm{p}<0.001$ ), while patients with $A F$ in the surgical intensive care unit (SICU) had an increased risk of 90-day mortality (HR 1.30, 95\% CI 1.13-1.51, p <0.001).

\section{Discussion}

The incidence of new-onset AF is high in current critically ill patients and is associated with an increased mortality rate. However, strategies for treatment and management of these patients remain controversial. In this large retrospective study, we provide an important 
Table 1 Baseline characteristics of the study population

\begin{tabular}{|c|c|c|c|c|}
\hline Variable & $\begin{array}{l}\text { Non-AF } \\
(n=28,802)\end{array}$ & $\begin{array}{l}\text { New-onset AF } \\
(n=3605)\end{array}$ & $\begin{array}{l}\text { Preexisting AF } \\
(n=5752)\end{array}$ & $p$ value \\
\hline Age(years) & $60.30 \pm 17.63$ & $72.96 \pm 11.75$ & $75.53 \pm 11.38$ & $<0.001$ \\
\hline Male(n(\%)) & $16,249(56.42)$ & $2162(59.97)$ & $3186(55.39)$ & $<0.001$ \\
\hline \multicolumn{5}{|l|}{ Comorbidities(n(\%)) } \\
\hline Hypertension & $11,911(41.35)$ & $1929(53.51)$ & 2691 (46.78) & $<0.001$ \\
\hline Diabetes & 7109 (24.68) & $1054(29.32)$ & $1648(28.65)$ & $<0.001$ \\
\hline $\mathrm{CHD}$ & 7691 (26.70) & $1857(51.51)$ & $2239(38.92)$ & $<0.001$ \\
\hline Congestive heart failure & $5054(17.55)$ & $1258(34.90)$ & $2883(50.12)$ & $<0.001$ \\
\hline Hyperlipidemia & $4399(15.27)$ & $894(24.80)$ & $1124(19.54)$ & $<0.001$ \\
\hline COPD & $539(1.87)$ & $69(1.91)$ & $168(2.92)$ & $<0.001$ \\
\hline Previous cerebral infarction & $1246(4.33)$ & $253(7.02)$ & $525(9.13)$ & $<0.001$ \\
\hline Pulmonary embolism & $746(2.59)$ & $58(1.61)$ & $143(2.49)$ & 0.002 \\
\hline Sepsis & $2371(8.23)$ & $279(7.74)$ & 701 (12.19) & $<0.001$ \\
\hline Hypothyroidism & $2477(8.60)$ & 407 (11.29) & $686(11.93)$ & $<0.001$ \\
\hline Type of ICU (n(\%)) & & & & $<0.001$ \\
\hline $\mathrm{CCU}$ & $3918(13.60)$ & $545(15.12)$ & $1147(19.94)$ & \\
\hline CSRU & $4682(16.26)$ & $1624(45.05)$ & $1269(22.06)$ & \\
\hline MICU & $10,737(37.28)$ & $798(22.14)$ & $1990(34.60)$ & \\
\hline SICU & $5059(17.56)$ & $390(10.82)$ & $846(14.71)$ & \\
\hline TSICU & $4406(15.30)$ & $248(6.88)$ & $500(8.70)$ & \\
\hline \multicolumn{5}{|l|}{ Initial laboratory data } \\
\hline WBC, $10^{3} / \mathrm{uL}$ & $11.74 \pm 5.80$ & $12.26 \pm 5.68$ & $12.07 \pm 5.94$ & $<0.001$ \\
\hline Hemoglobin, mg/dl & $11.06 \pm 1.99$ & $10.44 \pm 1.90$ & $10.70 \pm 1.92$ & $<0.001$ \\
\hline Creatinine, mg/dl & $1.2(0.7-1.4)$ & $1.2(0.8-1.4)$ & $1.4(0.8-1.6)$ & $<0.001$ \\
\hline SOFA score & $3.7(2-6)$ & $4.6(2-6)$ & $4.2(2-6)$ & $<0.001$ \\
\hline \multicolumn{5}{|c|}{ Rhythm or rate control drugs(n(\%)) } \\
\hline$\beta$-blockers & $13,638(47.35)$ & $2661(73.81)$ & 4371 (75.99) & $<0.001$ \\
\hline Amiodarone & $882(3.06)$ & $1455(40.36)$ & $1807(31.42)$ & $<0.001$ \\
\hline Nondihydropyridine CCB & $978(3.40)$ & $423(11.73)$ & $1560(27.12)$ & $<0.001$ \\
\hline Digoxin & $318(1.10)$ & $243(6.75)$ & $926(16.10)$ & $<0.001$ \\
\hline Statin $(n(\%))$ & $5788(20.10)$ & $1459(40.47)$ & $1684(29.28)$ & $<0.001$ \\
\hline Warfarin $(n(\%))$ & $2204(7.65)$ & $1206(33.45)$ & $2501(43.48)$ & $<0.001$ \\
\hline
\end{tabular}

$A F$ atrial fibrillation, CHD coronary heart disease, COPD chronic obstructive pulmonary disease, CCU coronary care unit, CSRU cardiac surgery recovery unit, MICU medical intensive care unit, TSICU trauma/surgical intensive care unit, WBC white blood cell, SOFA sequential organ failure assessment, CCB calcium channel blocker

Table 2 Unadjusted outcomes of the study population

\begin{tabular}{|c|c|c|c|c|}
\hline & $\begin{array}{l}\text { Non-AF } \\
(n=28,802)\end{array}$ & $\begin{array}{l}\text { New-onset AF } \\
(n=3605)\end{array}$ & $\begin{array}{l}\text { Preexisting AF } \\
(n=5752)\end{array}$ & $\mathrm{p}$ value \\
\hline 90-day mortality(n(\%)) & $4394(15.26)$ & $751(20.83)$ & $1458(25.35)$ & $<0.001$ \\
\hline Hospital mortality(n(\%)) & $2742(9.52)$ & $481(13.34)$ & $861(14.97)$ & $<0.001$ \\
\hline Hospital LOS(days)mean(IQR) & $6.4(3.8-11.2)$ & $7.7(5.1-12.0)$ & $9(5.6-15.0)$ & $<0.001$ \\
\hline ICU LOS(days)mean(IQR) & $2.0(1.2-3.8)$ & $2.3(1.3-4.8)$ & $2.9(1.5-5.8)$ & $<0.001$ \\
\hline $\operatorname{AKI}(n(\%))$ & $11,036(38.3)$ & $1238(34.3)$ & $1803(31.3)$ & $<0.001$ \\
\hline
\end{tabular}

$A F$ atrial fibrillation, $L O S$ length of stay, IQR interquartile range, ICU intensive care unit, $A K I$ acute kidney injury

reference for the prognosis and management of newonset AF. After adjusting for confounding factors in two models using Cox and logistic regression analyses, new-onset AF was found to be associated with significantly higher 90-day and hospital mortality than non$\mathrm{AF}$ and preexisting $\mathrm{AF}$. The prevalence of new-onset $\mathrm{AF}$ 


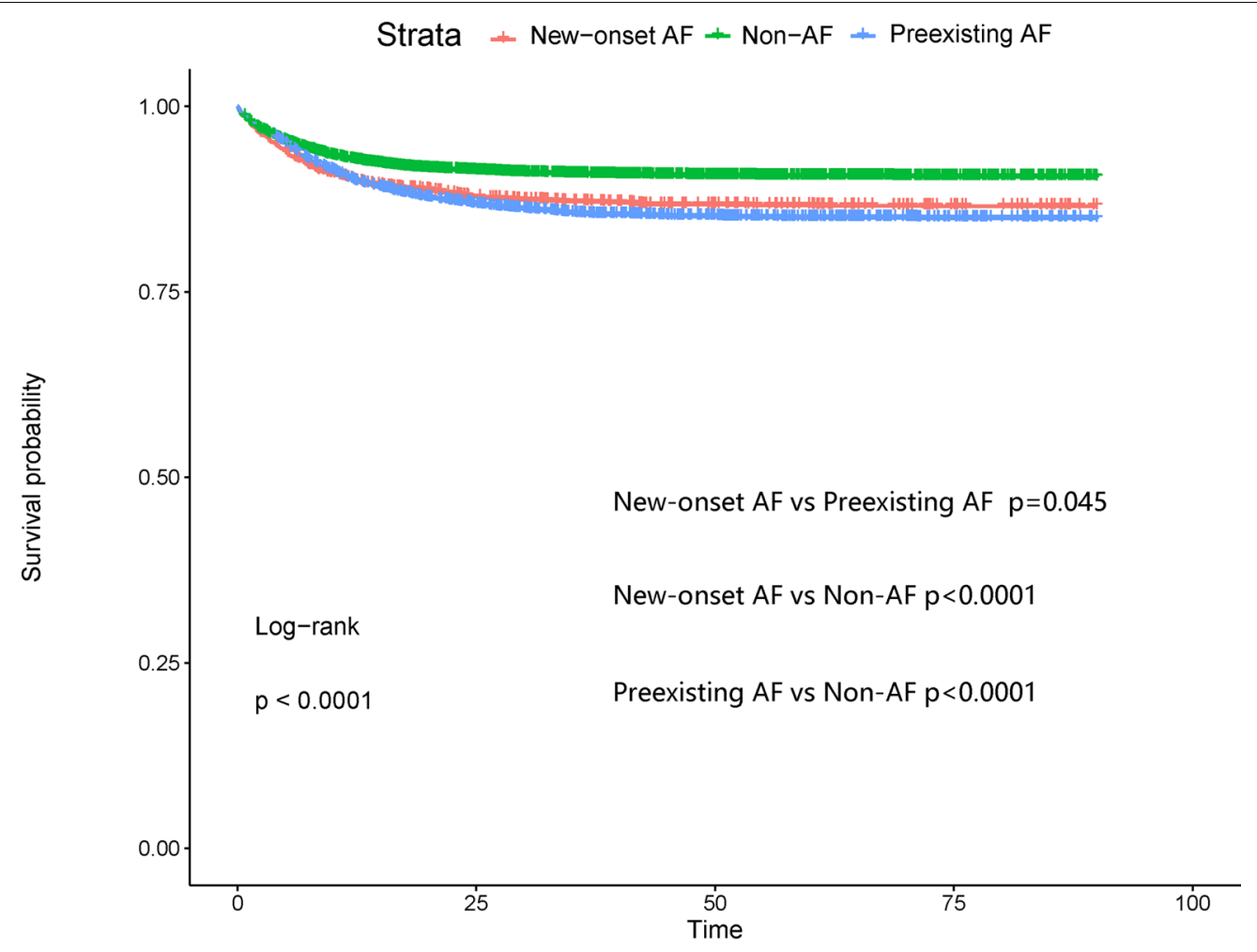

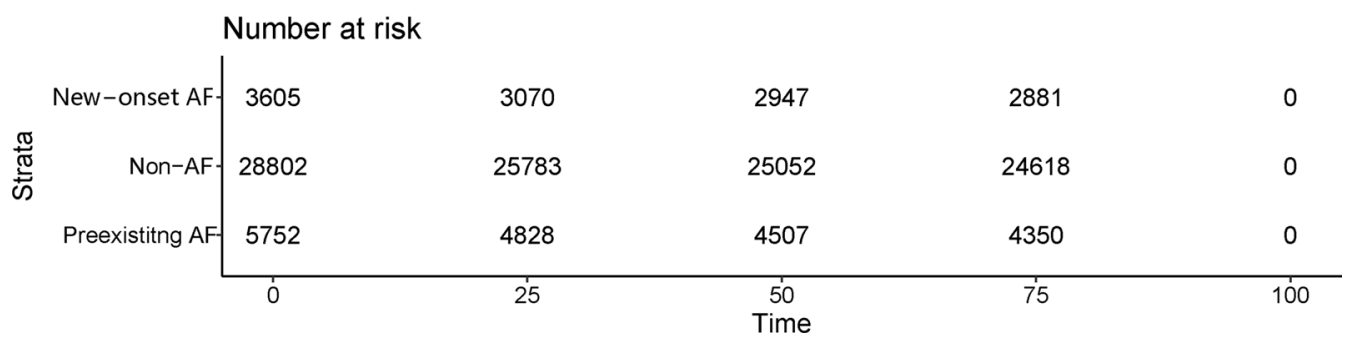

Fig. 2 Kaplan-Meier survival curve for the primary outcome of 90-day mortality in the 3 study groups. AF atrial fibrillation. Notes: Log-rank test $p<0.0001$

was approximately $9.4 \%$. Furthermore, after adjusting for confounding factors in model 2 using multivariate Cox analyses, we also demonstrated that age, congestive heart failure, cerebral infarction, pulmonary embolism, sepsis, SOFA score, WBC count, amiodarone use, non-dihydropyridine $\mathrm{CCB}$ use, and digoxin use were associated with an increased risk of 90-day mortality in patients with AF, while hypertension, hyperlipidemia, $\beta$ blockers use, statin use, warfarin use were protective factors for 90-day mortality in patients with AF. Patients with AF in the SICU had an increased risk of 90-day mortality compared with those in the CCU after adjusting for confounding variables.

Several previous studies have indicated that preexisting $\mathrm{AF}$ is associated with worse outcomes including higher rates of hospital and long-term mortality than nonAF patients $[4,9,13]$. In a previous retrospective study, preexisting AF was associated with an approximately 4 times increased risk of mortality compared with nonAF [20]. However, the effect of new-onset AF on mortality among critically ill patients remains controversial. Some prior studies did not support a significant correlation between new-onset AF and a high mortality risk $[15,21]$. Another prospective cohort study showed that new-onset or preexisting AF was independently associated with increased mortality [14]. In our study, the univariate Kaplan-Meier method showed that the 90-day mortality rate among patients with preexisting AF was higher than that among those with new-onset and nonAF. However, consistent with previous research, after adjustment using two different models, new-onset AF was associated with higher 90-day mortality than preexisting and non-AF. This may be because multiple potential factors affect mortality associated with new-onset 
Table 3 Adjusted hazard ratio of 90-day mortality comparing new-onset $A F$, non-AF and preexisting $A F$

\begin{tabular}{lllll}
\hline Outcomes & Group & Hazard ratio & $\mathbf{9 5 \%} \mathbf{C l}$ & p value \\
\hline $\begin{array}{llll}\text { 90-days mortality } \\
\text { Crude }\end{array}$ & Non-AF & Ref & & \\
& New-onset AF & 1.41 & $1.30-1.52$ & $<0.001$ \\
Model 1 & Non-AF & Ref & & \\
& New-onset AF & 1.33 & $1.22-1.44$ & $<0.001$ \\
Model 2 & Non-AF & Ref & & \\
& New-onset AF & 1.37 & $1.26-1.50$ & $<0.001$ \\
Crude & Pre-existing AF & Ref & & \\
& New-onset AF & 0.81 & $0.75-0.89$ & $<0.001$ \\
Model 1 & Pre-existing AF & Ref & & \\
& New-onset AF & 1.21 & $1.11-1.33$ & $<0.001$ \\
Model 2 & Pre-existing AF & Ref & & \\
& New-onset AF & 1.12 & $1.02-1.23$ & 0.019 \\
\hline
\end{tabular}

Model 1 was adjusted by: age, gender, type of intensive care unit, hypertension, diabetes, coronary heart diseases, congestive heart failure, hyperlipidemia, chronic obstructive pulmonary disease (COPD), cerebral infarction, pulmonary embolism, sepsis, and hypothyroidism; Model 2 was adjusted by: except variables in model 1, the following have been added: Sequential Organ Failure Assessment(SOFA) score, laboratory tests performed on admission, including white blood cells (WBC), hemoglobin (HB), serum creatinine ( $\mathrm{Scr}$ ), and medications during hospitalization, including $\beta$ blockers, statin, amiodarone, Non-dihydropyridine calcium channel blocker (CCB), digoxin, warfarin $A F$ atrial fibrillation, Ref reference

Table 4 Adjusted odds ratio of hospital mortality comparing new-onset $A F$, non-AF and preexisting AF

\begin{tabular}{lllll}
\hline Outcomes & Group & Odds ratio & $\mathbf{9 5 \%} \mathbf{C l}$ & p value \\
\hline $\begin{array}{llll}\text { Hospital mortality } \\
\text { Crude }\end{array}$ & Non-AF & Ref & & \\
& New-onset AF & 1.46 & $1.32-1.62$ & $<0.001$ \\
Model 1 & Non-AF & Ref & & \\
& New-onset AF & 1.46 & $1.30-1.64$ & $<0.001$ \\
Model 2 & Non-AF & Ref & & \\
& New-onset AF & 1.61 & $1.41-1.85$ & $<0.001$ \\
Crude & Preexisting AF & Ref & & \\
& New-onset AF & 0.88 & $0.77-0.99$ & 0.029 \\
Model 1 & Preexisting AF & Ref & & \\
& New-onset AF & 1.31 & $1.15-1.49$ & $<0.001$ \\
Model 2 & Preexisting AF & Ref & & \\
& New-onset AF & 1.17 & $1.01-1.35$ & 0.034 \\
\hline
\end{tabular}

Model 1 was adjusted by: age, gender, type of intensive care unit, hypertension, diabetes, coronary heart diseases, congestive heart failure, hyperlipidemia, chronic obstructive pulmonary disease (COPD), cerebral infarction, pulmonary embolism, sepsis, and hypothyroidism; Model 2 was adjusted by: except variables in model 1, the following have been added: Sequential Organ Failure Assessment(SOFA) score, laboratory tests performed on admission, including white blood cells (WBC), hemoglobin (HB), serum creatinine (Scr), and medications during hospitalization, including $\beta$ blockers, statin, amiodarone, Non-dihydropyridine calcium channel blocker (CCB), digoxin, warfarin $A F$ atrial fibrillation, Ref reference
AF. Nevertheless, prior studies have failed to compare the mortality risk between patients with new-onset AF and those with preexisting AF $[6,22,23]$. The prevalence of new-onset AF in ICU patients in the current study is comparable to that reported in previous research $[4,6$, $22,24]$. The reason why new-onset AF is associated with a poor prognosis is unclear. Infection and inflammation in critically ill patients may cause structural changes in the heart and accelerate electrical conduction $[25,26]$. In addition, patients with electrolyte imbalances and those being treated with vasopressin therapy are also more likely to develop $\operatorname{AF}[27,28]$. An observational study revealed that the incidence of hemodynamic instability in patients with new-onset AF was significantly higher than that in patients with preexisting AF [24]. During AF, coordinated depolarization and contraction of the heart are disturbed by countless and disordered atrial electrical pulses, resulting in unstable contractions and loss of "atrial rhythmic beating", thereby impairing cardiac output.

Notably, our study also demonstrated that age, congestive heart failure, cerebral infarction, pulmonary embolism, sepsis, SOFA score, WBC count, amiodarone use, non-dihydropyridine $\mathrm{CCB}$ use, and digoxin use were risk factors for 90-day mortality in critically ill patients with $\mathrm{AF}$, while hypertension, hyperlipidemia, $\beta$ blockers, statin and warfarin were protective factors with 90-day mortality (all $\mathrm{p}<0.05$ ). The incidence of previous cerebral infarction was significantly higher among patients with new-onset and preexisting AF than among patients with non-AF. Therefore, these patients may have had arrhythmic events prior to admission. However, we have no clear evidence to prove support this hypothesis. We focused on the effect of new-onset AF during hospitalization on 90 -day prognosis. The final conclusion may be biased. Development of AF during a critically ill period is related to the presence of more serious disease before the onset of AF and to clinical deterioration after AF; therefore, it is difficult to identify a causal role of AF in affecting patient prognosis. However, comparison of our findings with those of other studies confirms that elderly age, congestive heart failure, cerebral infarction, pulmonary embolism, and sepsis were associated with an obviously increased mortality risk in critically ill patients with $\operatorname{AF}[27,29]$. After evaluating the data, we found that the SOFA score in more than $90 \%$ of patients was less than 10 points. However, the SOFA scores we included were those obtained on admission, and not all patients were in a very critical condition at admission. Condition of patients may undergo a series of changes during their hospital stays. The distribution of data is shown in the Additional file 1: Fig. 1. We believe that these data are still representative of patients with severe illness. The 


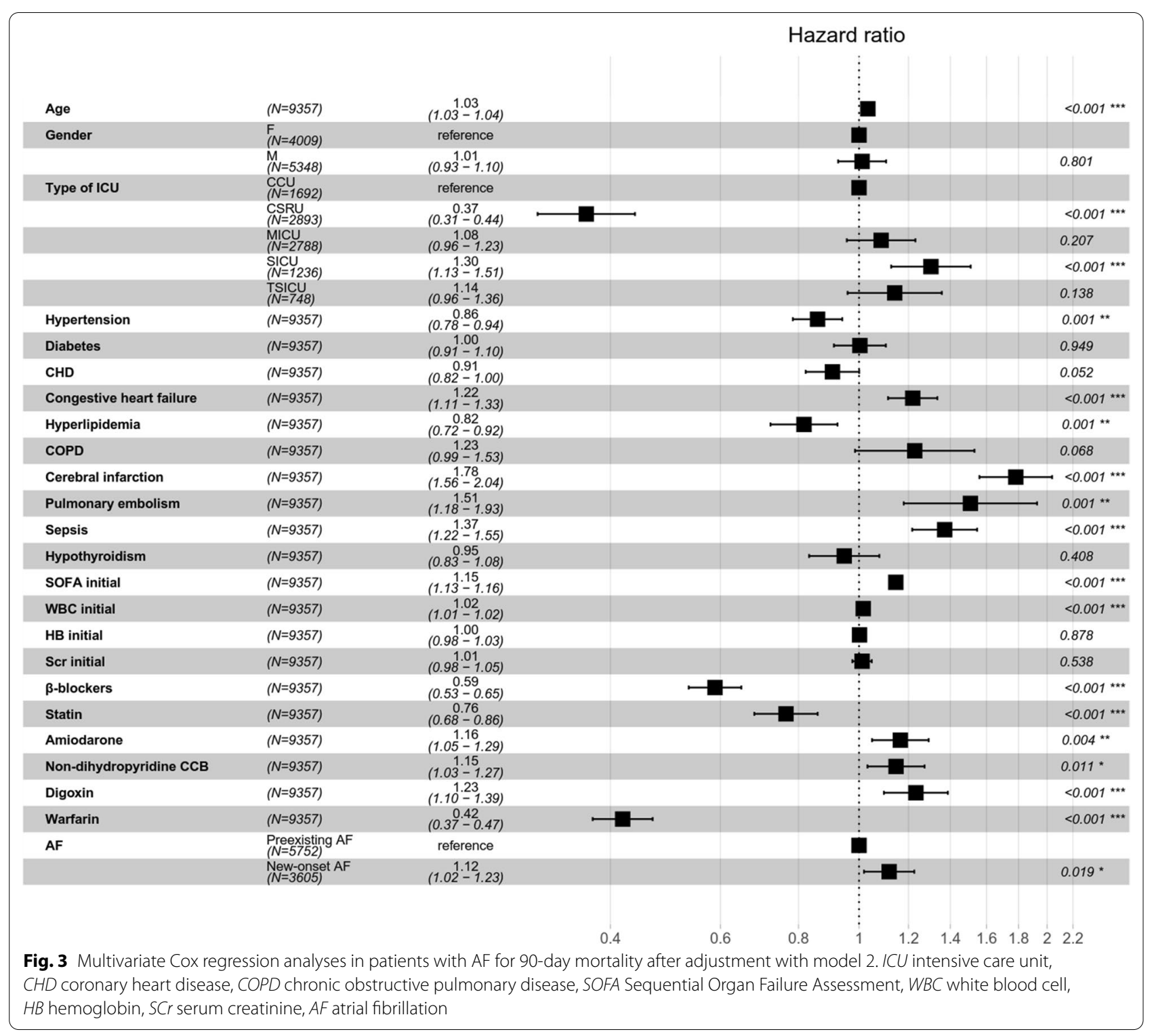

SOFA score was independently associated with 90-day mortality in patients with AF, which was also consistent with the founding of a previous study [30].

At present, there are still many controversies about the treatment strategy for patients with AF. Commonly used rate control drugs include non-dihydropyridine CCBs, $\beta$ blockers and digoxin. Rhythm control drugs usually include magnesium and amiodarone, both of which have rhythm and rate control properties. In the current study, we found that $\beta$ blockers, statins and warfarin were significantly associated with a decreased 90-day mortality risk, while amiodarone, non-dihydropyridine CCBs, and digoxin were associated with an increased risk. We evaluated the effect of warfarin on 90-day mortality in all patients with AF, including both those with newonset and preexisting AF. Warfarin was associated with a decreased 90-day mortality risk in patients with newonset and preexisting AF compared with those non-AF. $\beta$ blockers have rate control, negative muscle strength and vasodilatory effects. They exert sympathetic effects by antagonizing $\beta-1$ receptors, resulting in decreased conductivity and a reduced effect of catecholamines on the myocardium [31]. In accordance with the present results, a previous study demonstrated that $\beta$ blockers might be associated with lower hospital mortality in AF patients than amiodarone, non-dihydropyridine CCBs, and digoxin [32]. Digoxin slows the heart rate by increasing vagal nerve tension and may be related to hypotension. 
There is an association between digoxin and increased mortality, especially in patients with serum digoxin concentrations greater than $1.2 \mathrm{ng} / \mathrm{ml}$ [33]. In addition, the vagal nerve effects of digoxin may be less effective in severe diseases characterized by a high catecholamine status [34]. Amiodarone inhibits adrenergic stimulation, blocks delayed current, and slows atrioventricular conduction. However, this drug still has many shortcomings, such as hypotension, pro-arrhythmic effects, and pulmonary toxicity. In this study, we found that the most widely used antiarrhythmic drugs in patients with AF were $\beta$ blockers (75.2\%), amiodarone (34.9\%), CCBs (21.2\%), and digoxin (12.5\%). Among severely ill patients with AF in the United Kingdom, the most commonly used drug was amiodarone (>80\%), followed by $\beta$ blockers $(12 \%)$ [35]. Interestingly, in our study, statin use was also an independent protective factor for 90-day mortality in AF patients. This finding might be due to the anti-inflammatory effects of statins [36]. Patients with AF in SICU had increased 90-day mortality compared with those in the CCU after adjusting for confounding variables; thus, these patients may require more aggressive management.

\section{Limitations}

There are several study limitations of this study. First, due to the retrospective nature of this study, a causal relationship between new-onset AF and mortality cannot be directly inferred. Such a conclusion requires further research to establish a definitive causal link. Second, all patients with new-onset AF were diagnosed based on a well-defined 12-lead electrocardiogram, which is the clearest evidence. However, we did not have access to hourly cardiac monitoring information, which inevitably could have led to missing a proportion of patients with new-onset $\mathrm{AF}$, and to increasing the proportion of patients without AF. Nevertheless, an increased number of patients with non-AF would not significantly affect the results of this study. Third, although 2 different models were used to adjust for confounding factors, there may still be residual confounding factors that were not included. Finally, it is possible that health-care providers may choose pharmacological and electrical cardioversion during the onset of AF; this information was not available, which may have further increased the bias in this study.

\section{Conclusions}

In conclusion, after adjusting for confounding factors using Cox and logistic regression analyses, critically ill patients with new-onset AF had a significantly increased risk of hospital and 90-day mortality compared with those with preexisting AF and non-AF. Patients with AF in the CSRU had a decreased risk of 90-day mortality, while patients with AF in the SICU had an increased risk of 90-day mortality, compared with those hospitalized in the CCU. Management of patients with new-onset AF patients in the ICU, especially in the SICU, requires robust measures. $\beta$ blockers may be used as a first-line treatment for patients with AF in the ICU.

\section{Abbreviations}

AF: Atrialfibrillation; ICU: Intensivecare unit; MIMIC: Medicallnformation Mart for Intensive Care; SOFA: Sequentialorgan failure assessment; LOS: Lengthof stay; COPD: Chronicobstructive pulmonary disease; WBC: Whiteblood cells; HB: Hemoglobin; Scr: Serumcreatinine; CCB: Calciumchannel blocker; CSRU: Cardiacsurgical recovery unit; HR: Hazardratio; Cl: Confidenceinterval; CCU: Coronarycare unit; SICU: Surgicalintensive care unit.

\section{Supplementary Information}

The online version contains supplementary material available at https://doi. org/10.1186/s12872-021-02039-w.

Additional file 1. The distribution of SOFA score

Acknowledgements

Not applicable.

Authors' contributions

$J Q$ and FC: carried out the studies, participated in collecting data, and drafted the manuscript. LJK, LC and XBL: participated in collecting data and helped to draft the manuscript. JQ and LJK: performed the statistical analysis. $J Q$ and XBL: design, review and editing the manuscript. All authors read and approved the final manuscript.

\section{Funding}

This study was supported by the National Natural Science Foundation of China (Grant No. 81670403), Shanghai Science and Technology Committee (Nos. 18411950300, 19XD1403300, and 19411963200).

\section{Availability of data and materials}

The datasets used and analyzed during the current study are available from the corresponding author on reasonable request. The website of MIMIC database: https://mimic.physionet.org/.

\section{Declarations}

Ethics approval and consent to participate

The use of MIMIC database was approved by the Beth Israel Deaconess Medical Center (BIDMC) Institutional Review Board, therefore we did not require an ethical approval statement and informed consent for this study. Since any researcher who has completed the 'Protecting of Human Subjects' training can access the database, our data extraction processes were performed by Dr. Qian, following completion of the National Institutes of Health online training course (certificate code: 32299459).

Consent for publication

Not applicable.

Competing interests

The authors declare that they have no competing interests.

Author details

${ }^{1}$ Department of Cardiology, Shanghai Tongji Hospital, Tongji University School of Medicine, No.389, Xincun Rd, putuo District, Shanghai 200065, China.

${ }^{2}$ Department of Ultrasound, Luwan Branch, Ruijin Hospital, Shanghai Jiao Tong University School of Medicine, Shanghai, China. 
Received: 20 November 2020 Accepted: 21 April 2021

Published online: 05 May 2021

\section{References}

1. Pinho-Gomes AC, Amorim MJ, Oliveira SM, Leite-Moreira AF. Surgical treatment of atrial fibrillation: an updated review. Eur J Cardiothorac Surg 2014;46(2):167-78.

2. Calvo D, Filgueiras-Rama D, Jalife J. Mechanisms and drug development in atrial fibrillation. Pharmacol Rev. 2018;70(3):505-25.

3. January CT, Wann LS, Calkins H, Chen LY, Cigarroa JE, Cleveland JJ, et al. 2019 AHA/ACC/HRS Focused Update of the 2014 AHA/ACC/HRS Guideline for the Management of Patients With Atrial Fibrillation: A Report of the American College of Cardiology/American Heart Association Task Force on Clinical Practice Guidelines and the Heart Rhythm Society in Collaboration With the Society of Thoracic Surgeons. Circulation 2019:140(2):e125-51.

4. Bosch NA, Cimini J, Walkey AJ. Atrial fibrillation in the ICU. Chest 2018;154(6):1424-34

5. Moss TJ, Calland JF, Enfield KB, Gomez-Manjarres DC, Ruminski C, DiMarco JP, et al. New-onset atrial fibrillation in the critically III. Crit Care Med 2017;45(5):790-7.

6. Yoshida T, Fujii T, Uchino S, Takinami M. Epidemiology, prevention, and treatment of new-onset atrial fibrillation in critically ill: a systematic review. J Intensive Care 2015;3(1):19.

7. Aldhoon B, Melenovský V, Peichl P, Kautzner J. New insights into mechanisms of atrial fibrillation. Physiol Res 2010;59(1):1-12.

8. Ad N, Barnett SD, Haan CK, O'Brien SM, Milford-Beland S, Speir AM. Does preoperative atrial fibrillation increase the risk for mortality and morbidity after coronary artery bypass grafting? J Thorac Cardiovasc Surg 2009;137(4):901-6.

9. Rathore SS, Berger AK, Weinfurt KP, Schulman KA, Oetgen WJ, Gersh BJ, et al. Acute myocardial infarction complicated by atrial fibrillation in the elderly: prevalence and outcomes. Circulation 2000;101(9):969-74.

10. Foroulis CN, Kotoulas C, Lachanas H, Lazopoulos G, Konstantinou M, Lioulias AG. Factors associated with cardiac rhythm disturbances in the early post-pneumonectomy period: a study on 259 pneumonectomies. Eur J Cardiothorac Surg 2003;23(3):384-9.

11. Murthy SC, Law S, Whooley BP, Alexandrou A, Chu KM, Wong J. Atrial fibrillation after esophagectomy is a marker for postoperative morbidity and mortality. J Thorac Cardiovasc Surg 2003:126(4):1162-7.

12. Gialdini G, Nearing K, Bhave PD, Bonuccelli U, ladecola C, Healey JS, et al. Perioperative atrial fibrillation and the long-term risk of ischemic stroke. JAMA 2014;312(6):616-22.

13. Ruddox V, Sandven I, Munkhaugen J, Skattebu J, Edvardsen T, Otterstad JE. Atrial fibrillation and the risk for myocardial infarction, all-cause mortality and heart failure: a systematic review and meta-analysis. Eur J Prev Cardiol 2017;24(14):1555-66.

14. Shaver CM, Chen W, Janz DR, May AK, Darbar D, Bernard GR, et al. Atrial fibrillation is an independent predictor of mortality in critically ill patients. Crit Care Med 2015;43(10):2104-11.

15. Gupta S, Tiruvoipati R, Green C. Atrial fibrillation and mortality in critically ill patients: a retrospective study. Am J Crit Care 2015;24(4):336-41.

16. Reinelt P, Karth GD, Geppert A, Heinz G. Incidence and type of cardiac arrhythmias in critically ill patients: a single center experience in a medical-cardiological ICU. Intensive Care Med 2001:27(9):1466-73.

17. Annane D, Sébille V, Duboc D, Le Heuzey JY, Sadoul N, Bouvier E, et al. Incidence and prognosis of sustained arrhythmias in critically ill patients. Am J Respir Crit Care Med 2008;178(1):20-5.

18. Johnson AE, Pollard TJ, Shen L, Lehman LW, Feng M, Ghassemi M, et al. MIMIC-III, a freely accessible critical care database. Sci Data 2016;3:160035.
19. Kellum JA, Lameire N. Diagnosis, evaluation, and management of acute kidney injury: a KDIGO summary (Part 1). Crit Care 2013;17(1):204.

20. Lee E, Choi EK, Han KD, Lee H, Choe WS, Lee SR, et al. Mortality and causes of death in patients with atrial fibrillation: a nationwide population-based study. PLOS ONE 2018;13(12):e209687.

21. Meierhenrich R, Steinhilber E, Eggermann C, Weiss M, Voglic S, Bögelein $\mathrm{D}$, et al. Incidence and prognostic impact of new-onset atrial fibrillation in patients with septic shock: a prospective observational study. Crit Care 2010;14(3):R108.

22. Klein KP, Frencken JF, Kuipers S, Ong DS, Peelen LM, van Vught LA, et al. Incidence, predictors, and outcomes of new-onset atrial fibrillation in critically ill patients with sepsis. A cohort study. Am J Respir Crit Care Med 2017;195(2):205-11.

23. Arrigo M, Ishihara S, Feliot E, Rudiger A, Deye N, Cariou A, et al. New-onset atrial fibrillation in critically ill patients and its association with mortality: a report from the FROG-ICU study. Int J Cardiol 2018;266:95-9.

24. Kanji S, Williamson DR, Yaghchi BM, Albert M, Mclntyre L. Epidemiology and management of atrial fibrillation in medical and noncardiac surgical adult intensive care unit patients. J Crit Care 2012;27(3):321-6.

25. Korantzopoulos P, Kolettis TM. Obesity and the risk of new-onset atrial fibrillation. JAMA 2005;293(16):1974-5.

26. Mihm MJ, Yu F, Carnes CA, Reiser PJ, McCarthy PM, Van Wagoner DR, et al. Impaired myofibrillar energetics and oxidative injury during human atrial fibrillation. Circulation 2001;104(2):174-80.

27. Walkey AJ, Wiener RS, Ghobrial JM, Curtis LH, Benjamin EJ. Incident stroke and mortality associated with new-onset atrial fibrillation in patients hospitalized with severe sepsis. JAMA 2011;306(20):2248-54.

28. Walkey AJ, Greiner MA, Heckbert SR, Jensen PN, Piccini JP, Sinner MF, et al. Atrial fibrillation among Medicare beneficiaries hospitalized with sepsis: incidence and risk factors. Am Heart J 2013;165(6):949-55.

29. Chen AY, Sokol SS, Kress JP, Lat I. New-onset atrial fibrillation is an independent predictor of mortality in medical intensive care unit patients. Ann Pharmacother 2015;49(5):523-7.

30. Walkey AJ, McManus D. When rhythm changes cause the blues: new-onset atrial fibrillation during sepsis. Am J Respir Crit Care Med 2017;195(2):152-4.

31. Mareev Y, Cleland JG. Should $\beta$-blockers be used in patients with heart failure and atrial fibrillation? Clin Ther 2015;37(10):2215-24

32. Amsterdam EA, Kulcyski J, Ridgeway MG. Efficacy of cardioselective beta-adrenergic blockade with intravenously administered metoprolol in the treatment of supraventricular tachyarrhythmias. J Clin Pharmacol 1991:31(8):714-8

33. Rathore SS, Curtis JP, Wang Y, Bristow MR, Krumholz HM. Association of serum digoxin concentration and outcomes in patients with heart failure JAMA 2003;289(7):871-8.

34. Eisen A, Ruff CT, Braunwald E, Hamershock RA, Lewis BS, Hassager C, et al. Digoxin use and subsequent clinical outcomes in patients with atrial fibrillation with or without heart failure in the ENGAGE AF-TIMI 48 trial. J Am Heart Assoc 2017:6(7):e006035.

35. Chean CS, MCAuley D, Gordon A, Welters ID. Current practice in the management of new-onset atrial fibrillation in critically ill patients: a UK-wide survey. PeerJ 2017;5:e3716.

36. Jacob KA, Nathoe HM, Dieleman JM, van Osch D, Kluin J, van Dijk D. Inflammation in new-onset atrial fibrillation after cardiac surgery: a systematic review. Eur J Clin Investig 2014;44(4):402-28.

\section{Publisher's note}

Springer Nature remains neutral with regard to jurisdictional claims in published maps and institutional affiliations. 